ORIGINAL ARTICLE

\title{
Enhancing Personal Hygiene Behavior and Competency of Elementary School Adolescents through Peer-Led Approach and School-Friendly: A Quasi-Experimental Study
}

\author{
Dessalegn Tamiru $^{1 *}$, Alemayehu Argaw ${ }^{1}$, Mulusew Gerbaba ${ }^{1}$, Girmay Ayana ${ }^{2}$, \\ Aderajew Nigussie $^{1}$, Hunduma Jisha ${ }^{1}$, Tefera Belachew ${ }^{1}$
}

\section{OPEN ACCESS}

Citation: Dessalegn Tamiru, Alemayehu Argaw, Mulusew Gerbaba, Girmay Ayana, Aderajew Nigussie, Hunduma Jisha, Tefera Belachew. Enhancing Personal Hygiene Behavior and Competency of Elementary School Adolescents through Peer-Led Approach and School-Friendly: A QuasiExperimental Study. Ethiop J Health Sci 2017;27(3):245.

http://dx.doi.org/10.4314/ejhs.v27i3.6

Received: November 29, 2016

Accepted December 3, 2016

Published: May 1, 2017

Copyright: (c) 2017 Dessalegn T., et al.

This is an open access article distributed under the terms of the Creative Commons

Attribution License, which permits unrestricted use, distribution, and reproduction in any medium, provided the original author and source are credited.

Funding: Jimma University and

Ethiopian Public Health Institute

Competing Interests: The authors

declare that this manuscript was approved

by all authors in its form and that no

competing interest exists.

Affiliation and Correspondence:

${ }^{1}$ College of Health Science, Jimma University, Ethiopia

${ }^{2}$ Ethiopian Public Health Institute

*Email: dessalegn97@gmail.com

\begin{abstract}
BACKGROUND: Recent studies showed that poor personal hygiene practices play a major role in the increment of communicable disease burden in developing countries. In Ethiopia, $60 \%$ of the disease burden is related to poor sanitation practices. This school based study was aimed to assess the effectiveness of school-friendly and peer-led approach in improving personal hygiene practices of school adolescents in Jimma Zone, Southwest of Ethiopia.

METHODS: A total of 1000 students from 10 to 19 years were included into the study. The intervention was done using peer-led approach, health clubs and linking the school events with parents. Data were collected at baseline, midline and end-line using structured questionnaires. Repeated measurement analysis was done and statistical significance was considered at alpha 0.05 .

RESULTS: The findings of this study indicated that there was a significant difference in personal hygiene practices and knowledge between the intervention and control groups $(P<0.001)$. A significant difference was also observed with the duration of time in the intervention schools $(P<0.05)$. The proportion of adolescents who reported illness before the baseline survey was significantly high among the intervention schools $(P<0.01)$. However, at midline of the survey, the proportion of self-reported illness was significantly high among the control group $(P<0.001)$.

CONCLUSION: The findings of this study showed that there was a significant improvement in personal hygiene knowledge and practice of students in the intervention schools. Therefore, there is a need for proper health education intervention through the framework of schools for the students to improve their personal hygiene knowledge and practices.
\end{abstract}

KEYWORDS: Hygiene, School, Knowledge, Attitude, Jimma 


\section{INTRODUCTION}

Poor school sanitation and hygiene is a major problem in developing countries and remains high risk behaviour among primary school children. Over 1.9 billion school days could be gained if safe water supply and sanitation are achieved and the incidence of diarrhoeal illness is reduced $(1,2)$. The total global economic losses associated with inadequate water supply and sanitation was estimated at US\$ 260 billion annually (1).

Hygiene is a very personal subject, and encouraging changes in hygiene requires skill and care. The United Nations reported that there are 2.5 billion people who still do not use an improved sanitation facility and a little over 1 billion practising open defecation. Diarrhoea is the largest cause of under-five mortality globally in developing countries due to poor personal hygiene practices. In sub-saharan Africa, $44 \%$ of the population use either shared or unimproved facilities or an estimated $26 \%$ practice open defecation (2).

One of the target of Millennium Development Goal is to reduce by half the proportion of people without sustainable access to safe drinking water and basic sanitation by 2015 . However, the world remains off track to meet the target of $75 \%(1,2)$.

Health and health related problems are prevalent among school children, and adolescents in low income countries, partly due to their needs, have been largely ignored by the national policies, strategies and programs of the countries $(1,2,3)$. School children and adolescents are at their critical period in the lifetime that determines their current and future behaviors and risk factors related with health. If they are equipped with health promoting skills at this stage, they are more likely to adopt and sustain these behaviors throughout their lifetime (3-6). If knowledge is supported by enabling and reinforcing factors, desirable changes may occur in the school setting and in the community. Although the need for sanitation is widely known, reality does not reflect this perception. The gap between knowledge and practice is a common barrier to achieving health outcomes (1-4). A substantial body of evidence supports the effectiveness of school-based health education interventions on the development of the knowledge, attitudes, values and skills that are required to make the most appropriate and positive health-related decisions. School-based health education is recommended as one of the four pillars of focusing resources on effective school health (FRESH) that address priority health, hygiene and nutrition problems of school children and adolescents through the development of knowledge, attitudes and skills for promoting healthy behavior and lifestyle $(3,5)$.

Communicable diseases are considered as major causes of illness, death and disability in Ethiopia. In Ethiopia, $60 \%$ of the disease burden is related to poor sanitation and hygiene, and more than 250,000 children die every year from sanitation and hygiene related diseases (7).

In Ethiopia, National Strategy for Improved Hygiene and Sanitation has been developed to complement the existing health policy. However, the existing health information dissemination in schools mainly uses didactic approaches that lack a strong skill-building component. Success of school based education requires integration into the schools curricular and extracurricular activities with adequate time and intensity which do not exist in Ethiopian schools where health promotion activities are usually limited to special days and campaigns $(3,7,8,9)$.

Providing sanitation to people requires a systems approach, rather than only focusing on the toilet or waste water treatment plant itself. However, in Ethiopia there is scarcity of documented materials on the appropriateness and feasibility of school based interventions in improving personal hygiene practices. A wellorganized comprehensive school based personal hygiene and sanitation intervention is not yet integrated into the school curricula in Ethiopia. The limited practical experience and clear understanding on how to implement as well as the lack of evidence on the types of effective strategies for the country's setting are among the the factors that hinder the implementation of school based personal and environmental hygiene in Ethiopia $(1,7,10,11)$. Therefore, this study was aimed to assess the effectiveness of school based peer-led approach in improving personal hygiene

DOI: http://dx.doi.org/10.4314/ejhs.v27i3.6 
behavior and competency of school adolescents in Jimma Zone, Southwest Ethiopia.

\section{METHODS}

Study area and setting: This operational research was conducted from October 2013 to June 2014 in four primary schools of Jimma Zone. Jimma Zone is one of the 20 administrative zones of Oromia Regional State with its capital Jimma Town located at $350 \mathrm{~km}$ from Addis Ababa in the southwest direction. Jimma Zone has 18 administrative districts containing a total population of 2.5 million with the majority (94\%) living in rural settings. Studies from Jimma Zone showed that school children in the area are affected by different health problems (12).

Study design and sampling: A total of 1000 students were selected from 4 schools which were composed of 2 rural and 2 urban schools. The sample was calculated using Gpower 3.0 with the assumptions: $90.4 \%$ expected prevalence of poor diet and hygiene practices among school adolescents (13), margin of error 5\% and power of $88 \%$. This gives 434 sample size for both control and intervention groups and multiplied by design effect of two. Finally, 15\% of non-response was added to give a final sample size of 1000 .

Sampling was done separately for the control and intervention groups based on proportion to population size of the schools using a simple random sampling technique from each school. Data on outcome measures was collected at baseline, midline and the end-line from both students and their parents. A cohort of randomly selected students and their index mothers/ caretakers was followed up for a period of 8 months.

Intervention strategies: Intervention was done using a combination of strategies: demonstration, poster, role-play, peer to peer discussion, school media and health clubs. The intervention was implemented for a total of 8 months.

Situational analysis of needs and resources of the target group was done prior to the planning and implementation of the program. A detailed intervention protocol was developed based on formative assessment done at the beginning of the study. The formative assessment was done using exploratory qualitative research and on a desk review of available literature that shaped the detailed content of the study intervention, including the selection of behavioral models and preparation of education materials/resources.

Then, nutrition and health education specialists from Jimma University and Ethiopian Public Health Institute worked together on the design of education intervention including the selection of appropriate behavioral models, key messages and skills for promotion, development of health promotion materials and training modules. Then, the designed intervention was pilot-tested in two of the intervention schools (one urban and one rural) for two weeks, and final modification was done based on the result of the pilot testing.

Peer-led health education in the school was focused on general sanitation education concepts and skills. The peer educators were selected to teach students in group though demonstration and role-play. This was done once per two weeks. An informative meeting was done with the principal investigator and the supervisors to review peer educators role as educators, the importance of attendance for participation and its voluntary nature. The activity was aimed at developing the young children's knowledge, attitudes, beliefs and skills to enable them to be responsible for and to protect their own health. In addition, health messages promoting hygiene were also developed and posted on the walls given to peer-educators to refer to and deliver appropriate messages to group of students. In addition, teachers were expected to motivate and reward these peer groups where leaders acted as peer educators. The following procedure was followed to appropriately supervise the progress of the intervention with an assumption that a given students were exposed into multiple messages from first their intimate peer and then peer education led by peer educators for two days. Health messages developed in the local language were disseminated through minimedia. Teachers who received training of facilitation also guided and advised the students necessary. 


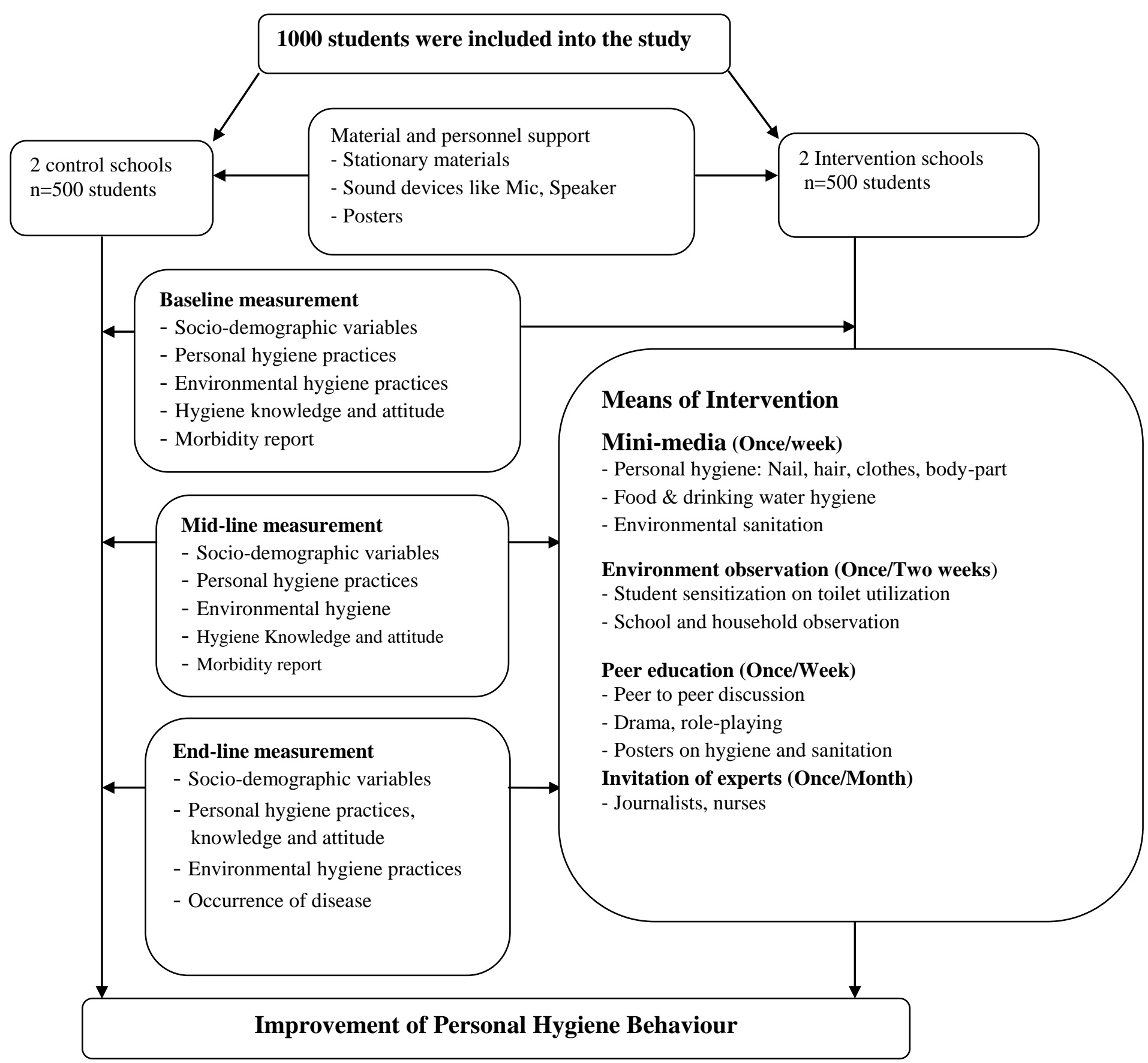

Figure 1: Conceptual framework of school intervention procedure in Jimma Zone, 2013.

Technical and material support was provided for intervention schools to strengthen/establish school health clubs and school media. Material support was also provided for control schools. In this intervention, the peer-led educations were further inspired by messages delivered using school media and health clubs. Audio records were also opened for students in Amharic and Afan Oromo

DOI: http://dx.doi.org/10.4314/ejhs.v27i3.6 
languages once a week. School club members, journalists and students working on school media were involved in delivering the key health and nutrition messages using artistic skills (role playing, posters, storytelling, debates, drama and audio-visual activities).

A committee was organized in each school with members from representatives of the school principal, unit leaders, club and peer-leaders. The committee had periodic meetings to identify and prioritize sanitary and other health problems and to create awareness in the community about the school-based health and nutrition education.

Data collection and analysis: Data were gathered using structured questionnaires and in-depth interviews of school principals, students and relevant teachers, observation of the school environment (water, sanitation, playing fields, available health services, school clubs and other extra-curricular activities), review of schools curricula and their health and nutrition policy. Data were collected at baseline, midline and endline of the study by trained research assistants. Data on socio-economic variables, the primary and secondary outcome measures were gathered using structured questionnaire through interview of children and their mothers/primary caretakers in the local language. In-depth interviews of school principals, students and relevant teachers and observation of the school environment were done to identify the appropriate intervention strategies.

To avoid the possibility of measurement bias, data collectors were blinded of the study objective and hypothesis. The items to assess knowledge, attitude and practice were developed based on the key messages promoted during the intervention. Morbidity status of children was assessed by caretakers' reported illness for the last two weeks. Monitoring of intervention implementation was conducted by supervisory visits to schools and performance evaluation meeting with the school principal, teachers facilitating the peer-education, heads of school media and health clubs. Periodic field visits were made by the research team. The research team also had periodic meetings to assess the progress and discuss necessary adjustments.

Before entry, data were coded and cleaned for consistency and completeness. Data were analysed using SPSS for windows version 16.0. Effectiveness of the intervention was assessed by comparing intervention and control groups at different points of intervention. To account for the effect of time trend, difference was measured using repeated measure analysis. All tests were two-sided and statistical significance was considered at alpha 0.05 . Multicollinearity among independent variables was detected using variance inflation factor showing that there was no multicollinearity $(\mathrm{VIF} \leq 10)$. Principal components analyses were used to generate composite score for practice, knowledge and attitude variables based on individual questionnaire items.

Ethical Consideration: Ethical approval of the study was granted from the Ethical Review Board of Jimma University, College of Public Health and Medical Sciences. Before the commencement of the intervention, a meeting was arranged with parents to explain about the purpose and nature of the study to obtain their consent.

\section{RESULTS}

Both intervention and control schools had much similar social and demographic characteristics except maternal marital status and educations level differences. At baseline, the mean age of students $( \pm \mathrm{SD})$ was $13.55( \pm 1.99)$ years (Table 1$)$.

Baseline data showed that there was no significant difference in personal hygiene practices between control and intervention groups except drinking water from well-protected sources, hand washing before handling and eating food (Table 2). 
Table 1: Socio-demographic characteristics of study participants at baseline in primary school of Jimma Zone, 2013

\begin{tabular}{|c|c|c|c|c|}
\hline Variables & Categories & $\begin{array}{l}\text { Intervention } \\
\text { group }(\%)\end{array}$ & $\begin{array}{l}\text { Control } \\
\text { group }(\%)\end{array}$ & P-value \\
\hline \multirow[t]{2}{*}{ Students' age } & $\leq 14$ years & $346(71.5)$ & $357(74.1)$ & \multirow{2}{*}{0.337} \\
\hline & $\geq 15$ years & $138(28.6)$ & $125(25.9)$ & \\
\hline \multirow[t]{2}{*}{ Students' sex } & Male & $179(49.2)$ & $185(50.8)$ & \multirow{2}{*}{0.657} \\
\hline & Female & $318(50.6)$ & $310(49.4)$ & \\
\hline \multirow[t]{4}{*}{ Students' grade } & Grade 5 & $146(150)$ & $146(50)$ & \multirow{4}{*}{0.267} \\
\hline & Grade 6 & $134(49.8)$ & $135(50.2)$ & \\
\hline & Grade 7 & $103(45.8)$ & $122(54.2)$ & \\
\hline & Grade 8 & $114(55.3)$ & $92(44.7)$ & \\
\hline \multirow{2}{*}{ Place of residence } & Urban & $256(50.7)$ & $249(49.3)$ & \multirow{2}{*}{0.704} \\
\hline & Rural & $241(49.5)$ & $246(50.5)$ & \\
\hline \multirow[t]{4}{*}{ Mothers' age } & $\leq 29$ & $23(39)$ & $36(61)$ & \multirow{4}{*}{0.013} \\
\hline & $\overline{30}-34$ & $77(54.2)$ & $65(45.8)$ & \\
\hline & $35-39$ & $145(45.2)$ & $176(54.8)$ & \\
\hline & $\geq 40$ & $153(56)$ & $120(44)$ & \\
\hline \multirow[t]{3}{*}{ Maternal marital status } & $\overline{\text { Father }}$ & $423(87.2)$ & $389(79.2)$ & \multirow[t]{3}{*}{0.004} \\
\hline & Mother & $20(4.1)$ & $47(9.6)$ & \\
\hline & Relatives & $42(8.6)$ & $55(11.1)$ & \\
\hline \multirow[t]{2}{*}{ Type of birth } & Single & 487(49.9) & $489(50.1)$ & \multirow{2}{*}{0.317} \\
\hline & Twin & $10(62.5)$ & $6(37.5)$ & \\
\hline \multirow[t]{2}{*}{ Family size } & $\leq 4$ & $100(24.0)$ & $121(28.3)$ & \multirow[t]{2}{*}{0.156} \\
\hline & $\geq 5$ & $317(76.0)$ & $307(71.7)$ & \\
\hline \multirow[t]{2}{*}{ Number of $\leq 5$ children } & $\overline{1}$ & $335(87.7)$ & $441(91.3)$ & \multirow[t]{2}{*}{0.083} \\
\hline & $\geq 2$ & $47(12.3)$ & $42(8.7)$ & \\
\hline \multirow[t]{3}{*}{ Religion } & Orthodox & $119(24.6)$ & $117(23.8)$ & \multirow{3}{*}{0.202} \\
\hline & Muslim & $307(63.6)$ & $332(67.6)$ & \\
\hline & Protestant, Wakefata & $57(11.8)$ & $42(8.5)$ & \\
\hline \multirow[t]{3}{*}{ Ethnicity } & Oromo & $358(74.1)$ & $325(67.0)$ & \multirow{3}{*}{0.34} \\
\hline & Amhara & $49(10.1)$ & $54(11.1)$ & \\
\hline & Dawuro, Tigre, Gurage & $76(15.8)$ & $33(6.8)$ & \\
\hline \multirow[t]{5}{*}{ Maternal Educational } & No education & $126(26.5)$ & $184(37.8)$ & \multirow{5}{*}{0.001} \\
\hline & Read and write & $47(9.9)$ & $82(16.8)$ & \\
\hline & $1^{0}$ school $(1-8)$ & $224(47.1)$ & $141(29.0)$ & \\
\hline & $2^{0}$ school $(9-12)$ & $54(11.3)$ & $64(13.1)$ & \\
\hline & Collage and above & $25(5.3)$ & $16(3.3)$ & \\
\hline \multirow{4}{*}{ Types of family toilet } & Water flushed and VIP & $18(45)$ & $22(55)$ & \multirow{4}{*}{0.245} \\
\hline & Pit latrine with slab & $344(47.7)$ & $377(52.3)$ & \\
\hline & Pit latrine without slab & $100(57.8)$ & $73(42.2)$ & \\
\hline & No toilet & $7(70)$ & $3(30)$ & \\
\hline
\end{tabular}

DOI: http://dx.doi.org/10.4314/ejhs.v27i3.6 
Table 2: Personal hygiene practices at baseline among primary school students in Jimma Zone, 2013

\begin{tabular}{lccr}
\hline Personal Hygiene Practices & $\begin{array}{c}\text { Intervention } \\
\text { Schools (\%) }\end{array}$ & $\begin{array}{c}\text { Control } \\
\text { Schools (\%) }\end{array}$ & P-value \\
\hline Wash hands with soap & $435(87.9)$ & $421(85.1)$ & 0.860 \\
Wash hands after toilet & $424(85.7)$ & $463(93.5)$ & 0.092 \\
Wear washed clothes & $141(28.5)$ & $89(18)$ & 0.155 \\
Walk barefoot & $104(21)$ & $90(18.2)$ & 0.262 \\
Brush teeth twice a day & $233(47.1)$ & $188(38)$ & 0.074 \\
Drink protected water & $421(85.2)$ & $439(88.7)$ & $<0.01$ \\
Always take a bath after playing & $256(51.7)$ & $216(43.6)$ & 0.091 \\
Cut nails weekly & $432(87.3)$ & $429(86.7)$ & 0.777 \\
Wash hands before handling food & $487(96.4)$ & $493(99.6)$ & $<0.01$ \\
Wash hands after eating food & $485(98)$ & $491(99.2)$ & $<0.01$ \\
Comb hair before going to school & $401(81)$ & $417(84.2)$ & 0.180 \\
Usually use toilet at home & $484(97.8)$ & $482(97.4)$ & 0.097 \\
Usually use toilet at school & $476(96.2)$ & $489(98.8)$ & 0.063 \\
Face is clean & $482(97.4)$ & $466(94.1)$ & 0.062 \\
Fingernails are trimmed & $126(25.5)$ & $302(61)$ & 0.067 \\
Eye discharge present & $451(91.1)$ & $393(79.4)$ & 0.061 \\
Hair is clean & $430(86.9)$ & $399(80.6)$ & 0.08 \\
\hline
\end{tabular}

The findings of this study showed that there was a significant difference in personal hygiene practice between the intervention and control schools $(\bar{x}=0.33, P<0.001)$. Among the control goup, there was only a significant difference from midline to baseline $(\mathrm{P}<0.01)$, but in case of the intervention group, a significant difference was observed over the follow-up period from midline to baseline $(<0.01)$ and end-line to baseline $(<0.001) \quad$ (Table $3)$.

Table 3: Personal hygiene practice differences by rounds and types of schools in Jimma Zone, 2013/14.

\begin{tabular}{|c|c|c|c|c|c|c|c|}
\hline \multirow{2}{*}{\multicolumn{3}{|c|}{ Comparison Group }} & \multirow{2}{*}{$\begin{array}{l}\text { Mean } \\
\text { Difference } \\
\end{array}$} & \multirow{2}{*}{$\begin{array}{l}\text { Std. } \\
\text { Error }\end{array}$} & \multirow[b]{2}{*}{ Sig. } & \multicolumn{2}{|c|}{$95 \%$ CI of Difference } \\
\hline & & & & & & Lower Bound & Upper Bound \\
\hline \multirow{3}{*}{ Control } & Midline(t2) & Baseline $\left(t_{1}\right)$ & 0.190 & 0.063 & $<0.01$ & 0.067 & 0.314 \\
\hline & End-line(t3) & Baseline $\left(t_{1}\right)$ & -0.022 & 0.065 & 0.732 & -0.150 & 0.105 \\
\hline & & Midline(t2) & -0.213 & 0.060 & 0.061 & 0.330 & -0.096 \\
\hline \multirow{3}{*}{ Intervention } & Midline(t2) & Baseline $\left(t_{1}\right)$ & $0.165^{*}$ & 0.054 & $<0.01$ & 0.059 & 0.270 \\
\hline & End-line(t3) & Baseline $\left(t_{1}\right)$ & $0.352^{*}$ & 0.053 & $<0.001$ & 0.248 & 0.457 \\
\hline & & Midline(t2) & $0.188^{*}$ & 0.048 & $<0.001$ & 0.093 & 0.282 \\
\hline School Type & Intervention & Control & 0.330 & 0.034 & $<0.001$ & 0.264 & 0.396 \\
\hline
\end{tabular}

Similar to personal hygiene practice, there was also a significant difference between intervention and control schools in personal hygiene knowledge $(\bar{x}=0.334, P<0.001)$ (Table 4). The

DOI: http://dx.doi.org/10.4314/ejhs.v27i3.6 
mean personal knowledge score was significantly increased among the intervention group over a follow up period and statistically a significant difference was observed between the intervention and control schools $(\mathrm{P}<0.05)$.

Table 4: Comprehensive personal hygiene knowledge between rounds and school type in Jimma Zone, $2013 / 14$

\begin{tabular}{|c|c|c|c|c|c|c|c|}
\hline \multirow{2}{*}{\multicolumn{3}{|c|}{ Comparison Group }} & \multirow{3}{*}{$\begin{array}{c}\begin{array}{c}\text { Mean } \\
\text { Difference }\end{array} \\
0.455^{*}\end{array}$} & \multirow{2}{*}{$\begin{array}{c}\text { Std. } \\
\text { Error }\end{array}$} & \multirow[b]{2}{*}{ Sig. } & \multicolumn{2}{|c|}{ 95\% CI for Difference } \\
\hline & & & & & & Lower Bound I & Lower Bound \\
\hline \multirow[t]{3}{*}{ Control } & Midline(t2) & Baseline $\left(\mathrm{t}_{1}\right)$ & & 0.071 & $<0.001$ & 0.314 & 0.595 \\
\hline & End-line(t3) & Baseline $\left(\mathrm{t}_{1}\right)$ & $0.432^{*}$ & 0.085 & 0.093 & 0.265 & 1.598 \\
\hline & & Midline(t2) & -0.023 & 0.077 & 0.767 & -0.174 & 0.128 \\
\hline \multirow[t]{3}{*}{ Intervention } & Midline(t2) & Baseline $\left(\mathrm{t}_{1}\right)$ & $0.733^{*}$ & 0.077 & $<0.001$ & 0.582 & 0.885 \\
\hline & End-line(t3) & Baseline $\left(\mathrm{t}_{1}\right)$ & $0.936^{*}$ & 0.080 & $<0.001$ & 0.779 & 0.993 \\
\hline & & Midline(t2) & $0.203^{*}$ & 0.045 & $<0.001$ & 0.113 & 0.292 \\
\hline School type & Intervention & Control & $0.344^{*}$ & 0.050 & $<0.001$ & 0.246 & 0.443 \\
\hline
\end{tabular}

*The mean difference is significant at the 0.05 level.

As illustrated in Figure 2, the proportion of adolescents who reported illness during the past two weeks before the baseline survey was significantly high among the intervention group (5\% vs $3 \%, \mathrm{P}=0.004)$. However, the subsequent follow-up showed that the proportion of self- reported illness was significantly higher among the controls $(7.87 \%)$ compared to intervention $(4 \%)$ schools at midline $(\mathrm{P}<0.0001)$. There was no statistically significant difference in morbidity between the two groups at the end-line survey $(\mathrm{P}=0.991)$.

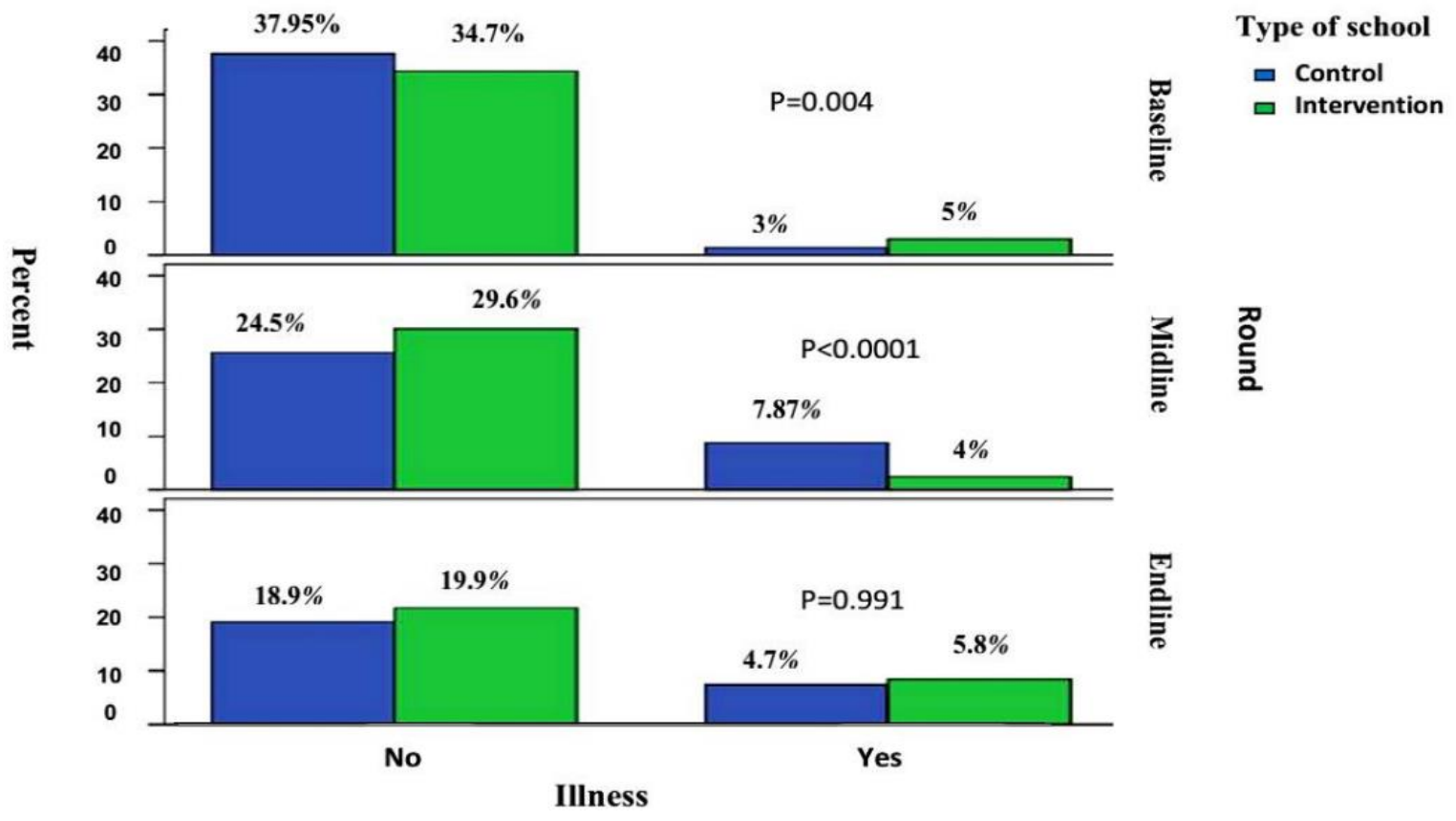

Figure 2: The proportion of self-reported illness during the past two weeks before survey among students by the round of study and intervention in Jimma Zone, 2013/14

DOI: http://dx.doi.org/10.4314/ejhs.v27i3.6 


\section{DISCUSSION}

The objective of the study was to investigate the impacts of health education intervention on personal hygiene behaviors and practices among the elementary school students in Jimma Zone. The findings of this study showed that there was a significant improvement in person hygine practices with the intervention period $(\mathrm{p}<0.05)$ among the intervention schools, but there was no significant change among the control group. This finding is consistent with studies done in India and other countries where personal hygiene knowledge plays a significant role in improving personal hygiene practices and attitudes (14-17). Studies from different parts of the world also showed that there was a significant difference in personal hygiene knowledge and practices with the duration of intervention $(14,15)$.

The findings of this study also indicated that there was a significant difference in personal hygiene knowledge between intervention and control groups at the end of the follow-up period $(\mathrm{P}<0.05)$. Overall, our findings are consistent with previous studies that documented knowledge and practices of hygiene among school children in developing countries (15-18). Studies also indicated that family socio-demographic variables like paternal literacy and sustained health education programs on personal hygiene in the school at regular intervals with greater involvement of benefit had significant inputs to improve the personal hygiene behavior of school adolescents (14).

The proportion of adolescents who reported illness during the past two weeks before the baseline survey was significantly higher among the intervention schools. However, at the mid-line survey, the proportion of students' self-reported illness increased in the control group and decreased in the intervention group $(\mathrm{P}<0.0001)$.
This might be due to seasonal variation which can causes outbreaks like diarrheal disease and malaria especially during the rainy season. However, there was no significant difference at end-line survey $(\mathrm{P}=0.991)$. Research review from developing countries similarly showed that health education had a significant input to sanitation behavior of school adolescents and their families. It has positive health consequences related to increased health education $(15,16)$. Other studies also showed that infectious diseases like diarrheal morbidity and behavoral change about personal hygiene are very personal subject, and encouraging changes in hygiene requires skill that can be improved through individual and community based education $(13,17)$. Generally, there was a significant improvement in the knowledge, attitude and practice level in study the schools compared to the control group. Therefore, there is a need for proper health education intervention through the framework of schools for the improvement of personal hygiene behavior of school adolescents and to reach the their families.

Although this study has a significant input for researchers and policy making, there is a limitation in that it did not include students from kindergarten and the first cycle primary school (Grade 1-4). Therefore, there is a need of further study on how to use these school students in improving their personal hygiene practices.

\section{ACKNOWLEDGEMENTS}

The authors would like to express their sincere gratitude to the Jimma University and Ethiopian Public Health Institute for funding the study and study participants for their diligence and dedication in collecting and inputting a high quality data used for the study. We are also thankful to the caretakers who gave their valuable time for the study.

LIST OF ABBREVIATIONS: AOR=Adjusted Odd Ratio, WHO=World Health Organization, $\mathrm{SD}=$ Standard Deviation

DOI: http://dx.doi.org/10.4314/ejhs.v27i3.6 


\section{REFERENCES}

1. United Nations Children's Fund (UNICEF): Child Friendly Schools Manual: Water, Sanitation and Hygiene (WASH) in Schools. Geneva, Switherland, 2012.

2. World Health Organization. Global costs and benefits of drinking-water supply and sanitation interventions to reach the MDG target and universal coverage. Geneva, Switzerland, 2012.

3. UNESCO/UNICEF/WHO/The World Bank. Focusing Resources on Effective School Health: A FRESH start to enhancing the quality and equity of education. World Education Forum 2000, Final Report. Dakar, Senegal, 2000.

4. Rodrigo C, Aranceta J. Nutrition education in schools: experiences and challenges. European Journal of Clinical Nutrition. 2003;57(1): S82-S85.

5. Rao G, Rao D, Venkaiah K, Dube A, Sarma K. Evaluation of the Food and Agriculture Organization's global school-based nutrition education initiative, Feeding Minds, Fighting Hunger (FMFH), in schools of Hyderabad. India Public Health Nutrition. 2006; 9(8):991995.

6. Peters L, Kok G, Ten Dam G. Effective elements of school health promotion across behavioral domains: a systematic review of reviews. BMC Public Health. 2009; 12(9):182-196.

7. Federal Democratic Republic of Ethiopia. Ministry of Health National Hygiene and Sanitation Strategy for Ethiopia. Addis Ababa, Ethiopia, 2010.

8. Centers for Disease Control and Prevention (CDC). Adolescent and school health. http://www.cdc.gov/nccdphp/dash/; 1999.

9. United Nations Children's Fund (UNICEF). Improving child nutrition the achievable imperative for global progress, United Nations Plaza. New York, USA, 2013.

10. World Health Organization. Sanitation and Hygiene Promotion: Programming Guidance. Geneva, Switzerland, 2005.
11. World Health Organization and United Nations Children's Fund. Joint Monitoring Programme for Water Supply and Sanitation (JMP). Progress on Drinking Water and Sanitation: Special Focus on Sanitation. Geneva, Switzerland, 2008.

12. Demissie F, Petros B, Kebede A. Hookworm species distribution among schoolchildren in Asendabo town, Jimma zone, southwest Ethiopia. Ethiopian Journal of Health Sciences. 2008; 18 (2):53-56.

13. Belachew T, Lindstrom D, Gebremariam A, Hogan D, Lacha, C, Huybregts L, Kolsteren P. Food insecurity, food based coping strategies and suboptimal dietary practices of adolescents in Jimma zone southwest Ethiopia. Plos One. 2013; 8(3):1-9.

14. Mukherjee A, Sinha A, Taraphdar P, Haldar D, Debasish S, Sinhal M. Effectiveness of an educational intervention on personal hygiene among school children in slum area of Kolkata, India. OSR Journal of Dental and Medical Sciences. 2014; 13(12):13-17.

15. Joshi A, Amadi C. Impact of Water, Sanitation, and Hygiene Interventions on Improving Health Outcomes among School Children. Journal of Environmental and Public Health Volume 2013. http://dx.doi.org/10.1155/2013/984626.

16. Fawole I, Asuzu M, Oduntan S, Brieger W. A school-based AIDS education programme for secondary school students in Nigeria: A review of effectiveness. Health Education research. 1999; 14(5): 675-683.

17. Mathai A, George S, Abraham J. Efficacy of a multimodal intervention strategy in improving hand hygiene compliance in a tertiary level intensive care unit. Indian Journal of Critical Care Medicine. 2011;15:1

18. Chola L, Michalow J, Tugendhaft A, Hofman K. Reducing diarrhoea deaths in South Africa: costs and effects of scaling up essential interventions to prevent and treat diarrhoea in under-five children. BMC Public Health. 2015; $15: 394$. 